\title{
The Mouse Mammary Video: An Introduction to the Mouse Mammary Gland and Instruction in its Surgical Manipulation
}

\author{
Charles W. Daniel • Phyllis Strickland
}

Published online: 12 August 2012

(C) Springer Science+Business Media, LLC 2012

Electronic supplementary material The online version of this article (doi:10.1007/s10911-012-9262-4) contains supplementary material, which is available to authorized users.

C. W. Daniel $(\bowtie) \cdot$ P. Strickland

Department of Molecular, Cell and Developmental Biology,

University of California, Santa Cruz,

Santa Cruz, CA 95064, USA

e-mail: gracedaniel@comcast.net

P. Strickland

e-mail: phyllis.strickland@gmail.com 